Instructions for authors, subscriptions and further details:

http://rasp.hipatiapress.com

\title{
Actividades de Ocio y Bienestar Emocional en Personas Jubiladas Independientes
}

Verónica López Fernández ${ }^{1}$, Enrique Navarro ${ }^{1}$, Carmen Requena Hernández ${ }^{2}$

1) Universidad Internacional de La Rioja

2) Universidad de León

Date of publication: July $30^{\text {th }}, 2015$

Edition period: July 2015 - January 2016

To cite this article: López, V., Navarro, E., \& Requena, C. (2015). Actividades de Ocio y Bienestar Emocional en Personas Jubiladas Independientes. Research on Ageing and Social Policy, 3(1), 46-63. doi: 10.17583/rasp.2015.1099

To link this article: http://doi.org/10.17583/rasp.2015.1099

\section{PLEASE SCROLL DOWN FOR ARTICLE}

The terms and conditions of use are related to the Open Journal System and to Creative Commons Attribution License (CCAL). 


\section{Actividades de Ocio y Bienestar Emocional en Personas Jubiladas Independientes}

Verónica López Fernández

Universidad Internacional de la Rioja

Carmen Requena Hernández

Universidad de León
Enrique Navarro

Universidad Internacional de la Rioja

\section{Resumen}

El propósito de este estudio es arrojar luz sobre la promoción del envejecimiento saludable y la calidad de vida de las personas mayores. Se ha comparado el bienestar emocional de una muestra de 56 personas sanas e independientes de la Provincia de León (España) en función del tipo de ocio que practican en su tiempo libre (actividades relacionadas con la formación, aulas de memoria y juegos de cartas). Se empleó la adaptación a España de la Escala de Bienestar Psicológico de Carol Ryff compuesta por seis dimensiones: dominio del entorno, autoaceptación, relaciones positivas con otras personas, autonomía, propósito de vida y crecimiento personal. Los resultados muestran que sólo existen diferencias estadísticamente significativas en la dimensión autonomía, favorables al grupo cuyo ocio predominante son tareas relacionadas con la mejora de la memoria frente al grupo de juegos de cartas. Estos resultados ponen de manifiesto la importancia de las actividades de ocio en el envejecimiento.

Palabras clave: bienestar emocional, ocio, envejecimiento, memoria 


\section{Leisure Activities and Emotional Well-being in Independent Retirees}

Verónica López Fernández

Universidad Internacional de la Rioja
Enrique Navarro

Universidad Internacional de la Rioja

Carmen Requena Hernández

Universidad de León

\section{Abstract}

This work aims to investigate the promotion of healthy aging and factors contributing on the quality of life of old people. A sample of 56 healthy, independent old people from the province of León (Spain) differing in the type of free time-entertainment (new information, memory and cards game) participated in this study. Emotional well-being state was evaluated using a Spanish-adaptation of the Carol Ryff Psychological Well Scale, composed of six dimensions: environmental mastery, self-acceptance, positive relations with others, autonomy, purpose of life and personal growth. Results show statistically significant differences in the Autonomy dimension, with favorable scores in those people who were predominantly involved in free time activities related to memory enhancement versus people who were predominantly involved in cards game. These findings highlight the relevance of the type of free time activities in elderly age, besides community activities.

Keywords: emotional well-being, free time, aging, memory. 
a perspectiva del curso vital está teniendo mucho auge en la investigación social (Mortimer y Shanahan, 2003) debido al gran número de personas de edad avanzada en las sociedades industrializadas con plena capacidad para la vida social (García y Viñado, 2013). En estas sociedades envejecidas, sigue vigente la perspectiva de la Organización Mundial de la Salud (OMS, 2002) que define la salud como un "estado de completo bienestar físico, mental y social, y no meramente la ausencia de enfermedad o dolencia". La salud ha de verse como un estado acumulativo que debe cuidarse a lo largo de toda la vida, con el fin de asegurarse el disfrute y los beneficios a edades avanzadas.

Brigeiro (2005) ha realizado un ensayo metódico y completo en el que reflexiona críticamente sobre el envejecimiento. Distingue el envejecimiento como proceso del proceso de envejecimiento. El envejecimiento como proceso ("envejecimiento normal") representa los cambios biológicos universales que se producen con la edad y no están afectados por la influencia de enfermedades o del entorno. Advierte que no todos estos cambios relacionados con la edad tienen consecuencias clínicas negativas. Por el contrario, el proceso de envejecimiento está muy influenciado por el entorno, el estilo de vida y las enfermedades que, a su vez, están relacionadas con el envejecimiento o cambian por su causa, pero que no se deben al envejecimiento en sí. A menudo, lo que una vez se consideró que era una consecuencia del proceso normal de envejecimiento se atribuye ahora, más apropiada-mente, a factores relacionados con el envejecimiento patológico. En determinados contextos, las definiciones comunes del envejecimiento raramente separan los dos procesos. Por ejemplo, el envejecimiento se ha definido como un "deterioro progresivo y generalizado de las funciones cognitivas, que produce una pérdida de respuesta adaptativa al estrés y un mayor riesgo de sufrir enfermedades relacionadas con la edad" (Kirkwood, 1996, citado en OMS, 2002, p. 86).

Los investigadores del envejecimiento se esfuerzan por definir un único concepto de envejecimiento: el exitoso. En este sentido, la OMS (2002), anima al "proceso de hacerse mayor sin envejecer mediante el desarrollo continuado de actividades físicas, sociales y espirituales a lo largo de toda la vida". En base a esta definición, hay muchos autores que argumentan que también la productividad laboral, de ocio u ocupacional son relevantes para potenciar el envejecimiento sano (Serrano, 2006). Envejecimiento exitoso, antes que un concepto, enmarca desde hace mucho una importante 
aspiración humana: deseamos envejecer bien, sin dolor o con el mínimo sufrimiento. Usualmente, miramos con admiración a aquellos que atraviesan los años revelando bienestar, realización personal, vivacidad y vigor preservados. Minkler y Fadem (2002) proponen un paradigma que contrasta con la asociación habitual de la etapa de la vejez y los procesos de pérdidas y deterioro. Se pretende formular una teoría que engendre aspectos positivos del envejecimiento. La primera característica a destacar en este estudio es la diferenciación realizada entre "envejecimiento exitoso" y "envejecimiento normal". El eje central de esta nueva perspectiva es la idea de que la salud y los problemas relativos al "envejecimiento normal" no son del todo normales, y más bien evidencian el resultado de estilos de vida y otros factores que expusieron a las personas al riesgo de enfermedades y discapacidades en la vejez.

En oposición, el "envejecimiento exitoso" es descrito como una habilidad para mantenerse en bajo riesgo de enfermar, con un alto nivel de actividad física y mental, y decididamente comprometido con la vida por medio del mantenimiento de relaciones interpersonales y la participación en actividades significativas. Dicho paradigma propone: o los temores de pérdidas funcionales son con frecuencia superestimados, o muchas de las pérdidas funcionales verificadas con el paso del tiempo pueden ser evitadas, o muchas pérdidas funcionales de los adultos mayores pueden ser recuperadas.

En este sentido, envejecer exitosamente dependería mayoritariamente de acciones en prevención y promoción de la salud, ya que la desdicha de la vejez asociada al deterioro físico y mental estaría, supuestamente, bajo nuestro control. Las pérdidas, según este paradigma, están asociadas a factores tales como hábitos cotidianos, alimentación, práctica de ejercicios, etc., todos ellos factibles de control y modificabilidad.

El envejecimiento exitoso, como afirman algunos autores, resulta ser una cuestión de opción individual (Kirkwood, 1996). Los modelos de envejecimiento exitoso en general, incluyendo el de la Fundación MacArthur ${ }^{1}$, pecan al generalizar premisas particulares y simples a contextos culturales diferenciados y complejos; aprisionan experiencias y valoraciones sociales diversas sobre el envejecer, bajo el auspicio de modelos estrechos y sin validación externa. Homogenizan prescripciones, sin matizar diferencias de género, raza y clase social, e ignoran las demás posibilidades que pueden caracterizar un buen envejecimiento. 


\section{López, Navarro \& Requena-Actividades de Ocio}

Brigeiro (2005) critica esta perspectiva de envejecimiento y recuerda que la edad por sí misma no justifica la normatividad de la experiencia. No todas las enfermedades y problemas fisiológicos asociados a la vida avanzada son factibles de manejo por medio de la prevención de la enfermedad o la promoción de la salud. Envejecer con salud requiere otras condiciones durante el curso de la vida, como el acceso a la educación, al trabajo y al descanso, bienes materiales y culturales (Debert, 1999). Hay que considerar la multiplicidad y los límites en el modo de envejecer como un acto de responsabilidad tanto del sujeto como del contexto social (Crawford, 1999).

Ya en 1961, autores como Neugarten, Havighurst y Tobin (1961) afirmaban que en la vejez, el bienestar se constituye como uno de los principales criterios para alcanzar un envejecimiento exitoso. Por todo ello, el hecho de conocer el bienestar de las personas mayores puede acercarnos a la realidad del proceso de envejecimiento. Sin embargo, esta valoración subjetiva de felicidad asociada a la edad ha sido muy poco estudiada. Tal y como señala Carmona (2009), desde hace tiempo se han estudiado los factores que intervienen en el bienestar y en el mantenimiento de una vida saludable. A principios de los años 80 , el concepto de bienestar empieza a verse como un constructo integrador de diferentes facetas de la vida (factores sociales, biológicos,...).

Autores relevantes como Ryff y Keyes (1995) otorgan importancia a la aceptación y crecimiento personal del sujeto, así como su adaptación e integración en su ambiente social, dando lugar a un concepto de bienestar integrador compuesto por las siguientes seis dimensiones que se describen a continuación: autoaceptación, relaciones positivas, autonomía, dominio del entorno, propósito en la vida y crecimiento personal. La autoaceptación es uno de los criterios centrales del bienestar. Las personas intentan sentirse bien consigo mismas incluso siendo conscientes de sus propias limitaciones. Tener actitudes positivas hacia uno mismo es una característica fundamental del funcionamiento psicológico positivo (Keyes, Ryff y Shmotkin, 2002). Lo es también la capacidad de mantener relaciones positivas con otras personas (Erikson, 1996; Ryff y Singer, 1998). La gente necesita mantener relaciones sociales estables y tener amigos en los que pueda confiar. La capacidad para amar es un componente fundamental del bienestar (Allardt, 1996) y consiguientemente de la salud mental (Ryff, 1989b). De hecho, numerosas investigaciones realizadas en las dos últimas décadas (Berkman, 1995; Davis, Morris y Kraus, 1998; House, Landis y Umberson, 1988) 
señalan que el aislamiento social y la pérdida de apoyo social están firmemente relacionados con el riesgo de padecer una enfermedad, y reducen el tiempo de vida. Otra dimensión esencial es la autonomía. Para poder sostener su propia individualidad en diferentes contextos sociales, las personas necesitan asentarse en sus propias convicciones (autodeterminación), y mantener su independencia y autoridad personal (Ryff y Keyes, 1995). Las personas con autonomía son capaces de resistir en mayor medida la presión social y auto-regulan mejor su comportamiento (Ryff y Singer, 2002). El dominio del entorno, es decir, la habilidad personal para elegir o crear entornos favorables para satisfacer los deseos y necesidades propias, es otra de las características del funcionamiento positivo. Las personas con un alto dominio del entorno poseen una mayor sensación de control sobre el mundo y se sienten capaces de influir sobre el contexto que les rodea. Finalmente, las personas sanas necesitan marcarse metas, definir una serie de objetivos que les permitan dotar a su vida de un cierto sentido. Necesitan, por tanto, tener un propósito en la vida. El funcionamiento positivo óptimo no sólo requiere de las características anteriormente señaladas; necesita también el empeño por desarrollar sus potencialidades, por seguir creciendo como personas y llevar al máximo sus capacidades, es decir, crecimiento personal (Keyes, Ryff y Shmotkin, 2002).

Diversos estudios transversales y longitudinales han intentado esclarecer el fenómeno de la "paradoja del bienestar en la vejez", referido a la presencia de índices de bienestar bastante elevados, a pesar de las dificultades, problemas o factores de riesgo (exclusión social, pérdida del nivel de ingresos, ...), habitualmente presentes en el contexto vital de algunas personas que, desde un punto de vista objetivo, harían hipotetizar infelicidad (Mroczek y Kolarz, 1998; Zamarrón, 2006) y que, sin embargo, muestran y manifiestan sentirse bien. Zamarrón (2006) argumenta que las explicaciones sobre este fenómeno podrían deberse a que las personas mayores afrontan mejor diferentes situaciones y circunstancias adversas, por lo que también se adaptarían mejor a las nuevas situaciones. Además, otra explicación podría ser que las personas a medida que envejecen, se marcan objetivos más realistas y fáciles de cumplir que las personas jóvenes.

Otro de los factores que se relaciona con el bienestar es la denominada eudaimonia, esto es, el resultado de estar implicado en un proceso de autorrealización y consecución de los valores personales que nos aportan significado y propósito en la vida (Ryan y Deci, 2001). Las personas 


\section{López, Navarro \& Requena-Actividades de Ocio}

mayores muestran niveles similares a los jóvenes y adultos de mediana edad en auto-aceptación, relaciones positivas y control sobre su entorno, si bien su nivel de crecimiento personal y propósito en la vida era significativamente menor (Ryff, 1989a). Se ha señalado que el declive en estos componentes del bienestar podría ser un reflejo de un proceso de adaptación a la etapa de la vejez, en la que la limitación del horizonte temporal hace que la proyección hacia el futuro no sea tan interesante como la focalización en el presente y el disfrute de lo conseguido hasta el momento (Carstensen, 1995; Ryff, 1989a; Villar, Triadó, Resano y Osuna, 2003).

\section{Actividades de Ocio y Bienestar Emocional}

Diversas investigaciones muestran los beneficios de la práctica de actividades de ocio en la contribución al bienestar psicológico y la satisfacción con la vida, propiciando una adaptación exitosa tras la jubilación (Nimrod, 2007). Sirva de ejemplo un estudio llevado a cabo por García y Gómez (2003) en el que se estudiaron los efectos psicológicos que tienen los talleres de actividades de ocio, que se llevan a cabo en un Centro de Día para Mayores, sobre el bienestar subjetivo de los 122 participantes, con edades comprendidas entre los 50 y 82 años, que realizaban actividades diferentes, agrupadas en: gimnasia, manualidades, informática, dibujopintura y control. Empleando un diseño cuasi-experimental pre-test/post-test, se analizaron las variables: satisfacción vital, depresión y soledad. Los resultados que encontraron confirman que dichas actividades de ocio hacen disminuir los niveles de depresión y soledad entre los participantes, al tiempo que ayudan a incrementar su satisfacción vital.

Merino y García (2005) resaltan la importancia social del envejecimiento en cuanto a la productividad y la reproductividad. Así, según estos autores en las sociedades occidentales, los valores se asientan sobre dichas capacidades, y a medida que se envejece los roles sociales se alejan de la esfera productiva (jubilación) y reproductiva (especialmente en las mujeres tras el climaterio). Todo ello lleva a una mayor disponibilidad de tiempo y a poseer menos responsabilidades, lo que requiere un gran esfuerzo de adaptación por parte de las personas mayores. Por ello, y según lo expuesto, las actividades de ocio pueden suponer una alternativa importante de cara a fomentar el bienestar emocional de las personas mayores. 
El presente estudio trata de responder a la siguiente cuestión: ¿existen diferencias en el bienestar psicológico en las personas jubiladas independientes en función del tipo de ocio que practican (ocio relacionado con la formación, tareas cognitivas y tareas procedimentales)? Para dar respuesta la pregunta, se marcan los siguientes objetivos: conocer el tipo de actividades de ocio que realizan las personas mayores sanas e independientes seleccionadas para esta investigación, así como conocer su bienestar psicológico; y cotejar si hay diferencias en la valoración del bienestar psicológico según el tipo de actividades de ocio que practican estas personas mayores.

La hipótesis de partida gira en torno a que el tipo de práctica de ocio diferenciará a los grupos en las medidas del bienestar psicológico, siendo mayor este bienestar en aquellos grupos que practican actividades denominadas cognitivas y de formación. Asimismo, el grupo de sujetos que se dedica a actividades de ocio relacionadas con actividades de formación tendrá mayor bienestar psicológico, que los que se dedican a actividades de ocio procedimentales.

\section{Metodología: Diseño, Muestra, Instrumentos y Procedimiento}

\section{Diseño}

Diseño cuasi-experimental, multicéntrico y transversal, que analiza la variable independiente (práctica de ocio en las personas mayores) en función de la variable dependiente emocional, a saber, bienestar psicológico en personas mayores sanas.

\section{Muestra}

La captación de los participantes se hizo entre personas mayores con patrones de comportamiento comunes: mayores de 56 años que viven de forma independiente, disponen de más de ocho horas de tiempo libre y acuden de forma habitual a centros de día de mayores del ayuntamiento de León capital y Ponferrada para participar en actividades de ocio. Se excluyeron los sujetos con solicitud de valoración de dependencia. Hemos tratado el concepto de tiempo libre como horas desocupadas de obligaciones externas y personales, es decir, el tiempo de ocio hace referencia a las 


\section{López, Navarro \& Requena-Actividades de Ocio}

obligaciones que se propone uno mismo en el tiempo libre. La muestra está formada por 56 personas que fueron asignados a tres grupos, en función de las actividades de ocio que practicaban (Tabla 1).

Tabla 1

Características de la Muestra

\begin{tabular}{|c|c|c|c|c|c|c|c|}
\hline \multirow{2}{*}{ Grupo } & \multirow{2}{*}{$\mathbf{N}$} & \multicolumn{2}{|c|}{ Sexo } & \multirow{2}{*}{$\begin{array}{l}\text { Edad } \\
\text { media }\end{array}$} & \multicolumn{3}{|c|}{ Estado civil } \\
\hline & & Mujeres & Hombres & & Casados & Solteros & Viudos \\
\hline G1 & 17 & 10 & 7 & 65 & 6 & 3 & 8 \\
\hline G2 & 15 & 8 & 7 & 74 & 10 & 1 & 4 \\
\hline G3 & 24 & 9 & 15 & 76 & 12 & 2 & 12 \\
\hline
\end{tabular}

\begin{tabular}{ccccccccc} 
& \multicolumn{3}{c}{ Estudios } & & \multicolumn{5}{c}{ Ocupación } \\
\cline { 2 - 3 } \cline { 7 - 9 } Grupo & Univ. & Bachill. & $\begin{array}{c}\text { Incom- } \\
\text { pletos }\end{array}$ & & $\begin{array}{c}\text { Funcio- } \\
\text { narios }\end{array}$ & $\begin{array}{c}\text { Comer- } \\
\text { cio }\end{array}$ & $\begin{array}{c}\text { Labores } \\
\text { labranza }\end{array}$ & $\begin{array}{c}\text { Lab. } \\
\text { hogar }\end{array}$ \\
\hline G1 & 5 & 3 & 9 & & 4 & 3 & 3 & 7 \\
G2 & 1 & 4 & 10 & & 1 & 1 & 4 & 9 \\
G3 & 3 & 3 & 18 & & 3 & 5 & 4 & 12 \\
\hline
\end{tabular}

En el grupo 1, actividad formativa, los participantes acuden a los programas de la Universidad de la Experiencia de la Universidad de León (en la sedes de León capital y Ponferrada). En dicho programa se imparten clases de Psicología, Derecho, Historia,... y se realizan circuitos culturales que organiza la Universidad. Dentro de este grupo se quedaron 17 participantes: 10 mujeres y 7 hombres, con una media de edad de 65 años.

Respecto al grupo 2, relacionado con aulas de memoria, los participantes realizan diferentes actividades mentales: cálculo, lectura, actividades de memoria, lenguaje,... Son actividades que realizan los CEAS (Centros de Acción Social) para mantener las funciones cognitivas. Dentro de este grupo 
se quedaron 15 participantes: 8 mujeres y 7 hombres, con una media de edad de 74 años.

En cuanto al grupo 3, juego de cartas, se caracteriza porque los participantes juegan en los centros para mayores (Hogar del Pensionista) a diferentes juegos de cartas, principalmente a la "brisca". Dentro de este grupo se quedaron 24 participantes: 9 mujeres y 15 hombres, con una media de edad de 76 años.

En la Tabla 1 puede verse el estado civil, el nivel de estudios y la ocupación de los participantes de la muestra. En todos los casos la evaluación y la participación en esta investigación se obtuvieron mediante consentimiento informado.

\section{Instrumentos: Escala de Bienestar Psicológico}

Es la versión española de la Escala desarrollada por Carol Ryff (1989a, 1989b). Dicha adaptación fue realizada por Díaz y cols. (2006). Esta versión desarrollada en español, se presenta como una buena herramienta para el estudio del bienestar psicológico en el campo de la psicología positiva desde el enfoque paradigmático de la felicidad eudaimónica desarrollado entre otros autores por Carol Ryff (1989a, 1989b) y Ryff y Singer (1998). El nuevo instrumento reduce la longitud de las escalas originales, facilitando su aplicación en diferentes ámbitos como la intervención social, la promoción de la salud, la gerontología o la psicoterapia, y, a su vez, mejora las propiedades psicométricas de la mayor parte de versiones existentes en inglés.

El instrumento mide el Bienestar Psicológico a través de un total de seis dimensiones (autoaceptación, relaciones positivas, autonomía, dominio del entorno, propósito en la vida y crecimiento personal). Consta de 39 ítems a los que los participantes deben responder utilizando un formato de respuesta tipo Likert con puntuaciones comprendidas entre 1 (totalmente en desacuerdo) y 6 (totalmente de acuerdo).

\section{Procedimiento}

La captación de la muestra se hizo en junio de 2013 en diez centros de día de León capital y Ponferrada en los que se proponen actividades de ocio para personas mayores. De entre las tres actividades más demandadas (formativa, 


\section{López, Navarro \& Requena-Actividades de Ocio}

curso de memoria o cognitiva y juegos de mesa o procedimental), se seleccionó la muestra de la investigación.

La divulgación de la investigación se llevó a cabo a través de una charla informativa acerca del objetivo del estudio y de las condiciones de participación. Los sujetos interesados cumplimentaron un informe con sus datos personales y posteriormente se contactó con ellos telefónicamente para las citas de evaluación. El tiempo de realización de las pruebas fue de 1 hora y 20 minutos. Los sujetos dieron y firmaron la hoja de consentimiento informado para la participación del estudio antes de las pruebas de evaluación. Se acordó con los sujetos de estudio la exposición de los resultados y conclusiones al finalizar la investigación.

\section{Resultados}

El tratamiento estadístico de los datos se realizó mediante el programa estadístico SPSS 17. A los datos obtenidos se les aplicó un análisis de varianza univariante (ANCOVA) de un factor considerando la variable dependiente cada dimensión de la escala y la independiente los factores inter-sujetos. Además se analizó el efecto de las covariables sexo y edad. En todos los casos se consideró un nivel de significación $\alpha \leq$. 05, y un IC (Intervalo de Confianza) para la media del 95\%. Se utilizó el método de contraste de Levene sobre la igualdad de las varianzas de error.

En relación a las seis dimensiones medidas en el Test de Bienestar Psicológico se obtuvieron los resultados siguientes en función de los grupos. Respecto a los datos descriptivos, las puntuaciones respecto a cada grupo pueden consultarse en la Tabla 2, así como los resultados comparativos.

Como puede apreciarse, sólo se han encontrado diferencias significativas en una dimensión del test empleado. Se ha encontrado diferencia estadísticamente significativa en la dimensión autonomía. Esta diferencia se ha hallado entre los grupos 2 y 3, a favor del G2 (p=.021). En la Tabla 2 pueden verse los resultados obtenidos en la variable grupo, así como otras variables como la edad y el sexo que podrían estar influyendo. Tras los análisis estadísticos, se ha constatado que la única diferencia obtenida se debe al efecto significativo del grupo. 
Tabla 2

Resultados comparativos entre grupos

\begin{tabular}{|c|c|c|c|c|c|c|c|}
\hline & \multirow[b]{2}{*}{$\mathrm{N}$} & \multicolumn{2}{|c|}{$\begin{array}{l}\text { Autoaceptación } \\
\text { (máx. 36) }\end{array}$} & \multicolumn{2}{|c|}{$\begin{array}{l}\text { Relac. Positivas } \\
\text { (máx. 36) }\end{array}$} & \multicolumn{2}{|c|}{$\begin{array}{c}\text { Autonomía } \\
\text { (máx. 48) }\end{array}$} \\
\hline & & $X$ & DT & $X$ & DT & $X$ & DT \\
\hline G1 & 18 & 26,71 & 2,95 & 24,47 & 5,76 & 32,00 & 5,33 \\
\hline $\mathrm{G} 2$ & 26 & 28,20 & 4,00 & 25,40 & 3,99 & 35,07 & 3,90 \\
\hline G3 & 28 & 28,79 & 7,52 & 23,04 & 6,02 & 30,04 & 8,05 \\
\hline
\end{tabular}

Datos comparativos:

$\begin{array}{lllc}\text { G1 vs G2 } & \text { n.s. } & \text { n.s. } & \text { n.s. } \\ \text { G1 vs G3 } & \text { n.s. } & \text { n.s. } & \text { n.s. } \\ \text { G2 vs G3 } & \text { n.s. } & \text { n.s. } & \mathbf{p = . 0 2 1}\end{array}$

\begin{tabular}{|c|c|c|c|c|c|c|c|}
\hline & \multirow[b]{2}{*}{$\mathrm{N}$} & \multicolumn{2}{|c|}{$\begin{array}{l}\text { Entorno } \\
\text { (máx. 36) }\end{array}$} & \multicolumn{2}{|c|}{$\underset{(\text { máx. 42) }}{\text { Crecimiento } P .}$} & \multicolumn{2}{|c|}{$\begin{array}{l}\text { Propósito V. } \\
\text { (máx. 36) }\end{array}$} \\
\hline & & $X$ & DT & $X$ & DT & $X$ & DT \\
\hline G1 & 18 & 26,35 & 4,31 & 30,76 & 4,20 & 26,71 & 3,17 \\
\hline $\mathrm{G} 2$ & 26 & 28,93 & 3,59 & 30,93 & 3,43 & 28,13 & 3,77 \\
\hline G3 & 28 & 27,83 & 7,82 & 32,63 & 8,03 & 30,04 & 7,12 \\
\hline
\end{tabular}

Datos comparativos:

\begin{tabular}{llll} 
G1 vs G2 & n.s. & n.s. & n.s. \\
G1 vs G3 & n.s. & n.s. & n.s. \\
G2 vs G3 & n.s. & n.s. & n.s. \\
\hline
\end{tabular}

En la Tabla 3 pueden verse los análisis de varianza univariante (ANCOVA) de las pruebas de los efectos inter-sujetos. Como puede verse, sólo la dimensión Autonomía tiene efecto significativo en función del grupo de ocio, efecto que no logran las covariables analizadas. En este caso, las diferencias en Autonomía se dan entre los grupos de cartas (puntuaciones más bajas, 26.52) y el grupo memoria (32.37, con las puntuaciones más altas). Esto quiere decir que las personas del grupo memoria, son significativamente más capaces de resistir en mayor medida la presión social y auto-regulan mejor su comportamiento que el grupo de cartas. 


\section{López, Navarro \& Requena-Actividades de Ocio}

\section{Tabla 3}

Análisis de varianza univariante (ANCOVA) de las pruebas de los efectos inter-sujetos en cada dimensión de bienestar

\begin{tabular}{|c|c|c|c|c|c|c|c|}
\hline Origen & $\begin{array}{c}\text { Suma de } \\
\text { cuadrados tipo III }\end{array}$ & gl & $\begin{array}{c}\text { Media } \\
\text { cuadrática }\end{array}$ & $\mathrm{F}$ & Sig. & $\begin{array}{l}\text { Parámetro de } \\
\text { no centralidad }\end{array}$ & $\begin{array}{c}\text { Potencia } \\
\text { observada }\end{array}$ \\
\hline \multicolumn{8}{|c|}{ Variable dependiente: Autoaceptación } \\
\hline Modelo corregido & $175,682^{\mathrm{a}}$ & 4 & 43,921 & 1,409 & ,244 & 5,635 & ,407 \\
\hline Intersección & 222,003 & 1 & 222,003 & 7,120 &, 010 & 7,120 & ,745 \\
\hline EDAD & ,133 & 1 & 133 &, 004 & ,948 & ,004 & ,050 \\
\hline SEXO & 75,600 & 1 & 75,600 & 2,425 & 126 & 2,425 & ,333 \\
\hline Grupo & 61,464 & 2 & 30,732 &, 986 & 380 & 1,971 & ,212 \\
\hline Error & 1590,157 & 51 & 31,180 & & & & \\
\hline Total & 44723,000 & 56 & & & & & \\
\hline Total corregida & 1765,839 & 55 & & & & & \\
\hline \multicolumn{8}{|c|}{ Variable dependiente: Relac. Positivas } \\
\hline Modelo corregido & $163,726^{a}$ & 4 & 40,931 & 1,409 & ,244 & 5,636 & ,407 \\
\hline Intersección & 183,820 & 1 & 183,820 & 6,327 &, 015 & 6,327 & 694 \\
\hline EDAD & ,251 & 1 & ,251 &, 009 & ,926 & ,009 & 051 \\
\hline SEXO & 109,024 & 1 & 109,024 & 3,753 & ,058 & 3,753 & 476 \\
\hline Grupo & 108,403 & 2 & 54,202 & 1,866 &, 165 & 3,731 & ,371 \\
\hline Error & 1481,632 & 51 & 29,052 & & & & \\
\hline Total & 34190,000 & 56 & & & & & \\
\hline Total corregida & 1645,357 & 55 & & & & & \\
\hline \multicolumn{8}{|c|}{ Variable dependiente: Autonomía } \\
\hline Modelo corregido & $345,840^{a}$ & 4 & 86,460 & 2,195 & ,083 & 8,779 & ,605 \\
\hline Intersección & 256,361 & 1 & 256,361 & 6,507 &, 014 & 6,507 & ,706 \\
\hline EDAD & 1,202 & 1 & 1,202 &, 031 & ,862 &, 031 & ,053 \\
\hline SEXO & 108,852 & 1 & 108,852 & 2,763 & ,103 & 2,763 & 371 \\
\hline Grupo & 328,301 & 2 & 164,150 & 4,167 &, 021 & 8,334 & ,709 \\
\hline Error & 2009,142 & 51 & 39,395 & & & & \\
\hline Total & 59635,000 & 56 & & & & & \\
\hline Total corregida & 2354,982 & 55 & & & & & \\
\hline \multicolumn{8}{|c|}{ Variable dependiente: Entorno } \\
\hline Modelo corregido & $101,431^{a}$ & 4 & 25,358 & ,703 &, 593 & 2,813 & ,212 \\
\hline Intersección & 208,100 & 1 & 208,100 & 5,772 & ,020 & 5,772 & 654 \\
\hline EDAD & , 000 & 1 & ,000 & ,000 & ,997 & ,000 & ,050 \\
\hline SEXO & 47,009 & 1 & 47,009 & 1,304 & ,259 & 1,304 & 202 \\
\hline Grupo & 59,099 & 2 & 29,550 & 820 &, 446 & 1,639 & 183 \\
\hline Error & 1838,784 & 51 & 36,055 & & & & \\
\hline Total & 44842,000 & 56 & & & & & \\
\hline Total corregida & 1940,214 & 55 & & & & & \\
\hline \multicolumn{8}{|c|}{ Variable dependiente: Crecim Personal } \\
\hline Modelo corregido & $90,561^{a}$ & 4 & 22,640 &, 613 & ,655 & 2,450 & ,188 \\
\hline Intersección & 500,050 & 1 & 500,050 & 13,531 & ,001 & 13,531 & ,950 \\
\hline EDAD & 42,348 & 1 & 42,348 & 1,146 & ,289 & 1,146 & 183 \\
\hline SEXO & 7,027 & 1 & 7,027 & 190 & ,665 & 190 & ,071 \\
\hline Grupo & 75,875 & 2 & 37,937 & 1,027 & ,366 & 2,053 & 219 \\
\hline Error & 1884,796 & 51 & 36,957 & & & & \\
\hline Total & 57920,000 & 56 & & & & & \\
\hline Total corregida & 1975,357 & 55 & & & & & \\
\hline \multicolumn{8}{|c|}{ Variable dependiente: Propósito Vida } \\
\hline Modelo corregido & $197,886^{\mathrm{a}}$ & 4 & 49,471 & 1,745 & ,155 & 6,979 & ,496 \\
\hline Intersección & 228,787 & 1 & 228,787 & 8,069 &, 006 & 8,069 & ,796 \\
\hline EDAD & ,034 & 1 & ,034 & ,001 & ,972 & 001 & ,050 \\
\hline SEXO & 83,787 & 1 & 83,787 & 2,955 &, 092 & 2,955 & ,392 \\
\hline Grupo & 49,804 & 2 & 24,902 & 878 & ,422 & 1,756 & ,193 \\
\hline Error & 1446,096 & 51 & 28,355 & & & & \\
\hline Total & 47187,000 & 56 & & & & & \\
\hline Total corregida & 1643,982 & 55 & & & & & \\
\hline
\end{tabular}




\section{Discusión}

Una vez analizados los datos y comprobado que las covariables edad y sexo no tienen efecto en los resultados encontrados, cabe comentar que los resultados del Test de Bienestar Psicológico que mide dimensiones tales como autoaceptación, relaciones positivas, propósito de vida y crecimiento personal muestran normalidad y similitud entre los tres grupos. Esto se explicaría en relación a que la organización de su tiempo de ocio no es impuesta, es la que quieren que sea. Heckhausen y Schulz (1995) sugieren dos tipos de control: primario y secundario que reajustan sus relaciones después de los 60 años. El control primario se caracteriza por la resistencia del sujeto para adaptarse a las exigencias del entorno, frente al secundario que se refiere a los cambios que realiza el sujeto sobre sí mismo para lograr sus intereses. Además, estos resultados están en consonancia con los obtenidos por Carmona (2009) que, en un estudio con 1056 personas mayores, encontró que uno de los factores que más se relaciona con el bienestar es la interacción social, que es el denominador común en los tres grupos de esta investigación. Podría decirse que la idiosincrasia de cada grupo de nuestra investigación dibuja un perfil distinto de envejecer, pero comparten la capacidad de controlar su vida y su entorno. Aún más, dado que la edad media de los sujetos del G2 y G3 se sitúa en torno a los setenta, cabría esperar declive en la autonomía (Willis y Marsiske, 1990), hecho que no se da en nuestra muestra. Esto nos lleva a confirmar los resultados de otras investigaciones que relacionan el mantenimiento de las AIVD con la práctica de ocio (Verghese y cols., 2003).

En cuanto a las diferencias obtenidas en la dimensión de autonomía entre los grupos 2 y 3, hemos encontrado que el grupo 3 muestra menor puntuación en esta dimensión, lo que podría indicar, siguiendo a Carmona (2009), que el grupo 3 vería reducida su capacidad funcional disminuyendo así su autonomía y optaría por actividades más cercanas. Es llamativo el hecho de que el grupo relacionado con información nueva (G1) no haya obtenido dichas diferencias con respecto al G3. Una posible explicación podría ser que, dado que la finalidad de las actividades de ocio radica en un mayor bienestar, dignidad y autonomía posible de las personas, puede ser importante tener en cuenta la autonomía previa de las personas (Serrano, 2006) y también del medio donde dichas personas residen. Probablemente, el grupo G1, al acudir a la Universidad para recibir las clases, se sienta menos 


\section{López, Navarro \& Requena-Actividades de Ocio}

autónomo que el G2, ya que debe coger autobuses urbanos y organizar con mayor planificación su ocio. En el G2, los participantes del estudio acuden a sus centros CEAS más cercanos para realizar el ocio que practican.

El hecho de no encontrar diferencias estadísticamente significativas en el resto de las dimensiones en función de los grupos de investigación estaría en consonancia con los hallazgos de García y Gómez (2003) que postulan que las actividades de ocio hacen disminuir los niveles de depresión y soledad entre los participantes, al tiempo que ayudan a incrementar su satisfacción vital y bienestar.

\section{Conclusiones}

La hipótesis de trabajo de este estudio era que el tipo de práctica de ocio diferenciaría a los grupos en las medidas del bienestar psicológico a favor de los que practican actividades denominadas cognitivas y de formación. Sólo se cumple parcialmente esta hipótesis en la dimensión autonomía, a favor del grupo de memoria frente al grupo de cartas. Los sujetos que se dedican a actividades de ocio relacionadas con la formación tendrán mayor bienestar psicológico que los que se dedican a actividades de ocio procedimentales. No se han encontrado diferencias significativas en las dimensiones del bienestar psicológico a favor del G1, relacionado con la formación.

Se requieren más investigaciones que ayuden a comprender con mayor nitidez la relevancia que tiene el tipo de ocio que se practica, en vías a minimizar el deterioro funcional y maximizar el bienestar de las personas mayores en contacto directo con el entorno.

\section{Notas}

${ }^{1}$ La Fundación MacArthur es una institución estadounidense que estudia el envejecimiento exitoso. Según esta Fundación, sólo el 30\% de las características del envejecimiento tienen un componente genético. El resto estarían bajo el control de cada individuo (Canet, 2005).

\section{Referencias}

Allardt, E. (1996). Tener, amar, ser: una alternativa al modelo sueco de investigación sobre el bienestar. En M. Nussbaum y A. Sen (comps.), La calidad de vida, México: F.C.E. (pp. 126-134). 
Berkman, L. F. (1995). The role of social relations in health promotion. Psychosomatic Medicine, 57(3), 245-254.

Brigeiro, M. (2005). "Envejecimiento exitoso" y "tercera edad": Problemas y retos para la promoción de la salud. Investigación y Educación en Enfermería - Medellín, 23(1), 102-109.

Canet, C. (2005). La tercera edad. Trafford Publishing.

Carmona, S. E. (2009). El bienestar personal en el envejecimiento.

IBEROFORUM: Revista de ciencias sociales de la Universidad Iberoamericana, 4(7), 48-65.

Carstensen, L. L. (1995). Evidence for a life-span theory of socioemotional selectivity. Current Directions in Psychol. Science, 4(5), 151-156 Crawford, R. (1999). Usted es peligroso para su salud: aspectos ideológicos y políticos de culpabilizar a la víctima. En: C. De la Cuesta (comp.), Salud y Enfermedad: lecturas básicas en sociología de la medicina. Medellín: Universidad de Antioquia (pp. 47- 77).

Davis, M. H., Morris, M. M. y Kraus, L. A. (1998). Relationship-specific and global perception of social support: Associations with well-being and attachments. Journal of Personality and Social Psychology, 74(2), 468-481. http://dx.doi.org/10.1037/0022-3514.74.2.468

Debert, G. A. (1999). A reinvenção da velhice: socialização e processos de reprivatização do envelhecimento. São Paulo: Universidade de São Paulo. FAPESP.

Díaz, D., Rodríguez-Carvajal, R., Blanco, A., Moreno-Jiménez, B., Gallardo, I., Valle, C. y van Dierendonck, D. (2006). Adaptación española de las escalas de bienestar psicológico de Ryff. Psicothema, 18(3), 572-577.

Erikson, R. (1996). Descripciones de la desigualdad: el enfoque sueco de la investigación sobre el bienestar. En M. Nussbaum y A. Sen (comps.), La calidad de vida. México: F.C.E. (pp. 101-120).

García, M. A. y Gómez, L. (2003). Efectos de los talleres de ocio sobre el bienestar subjetivo y la soledad en las personas mayores. Revista de Psicología social, 18(1), 35-47.

García, B. y Viñado, C. (2013). Análisis crítico del curso vital. Research on ageing and social policy, 1(1), 78-97. doi: 10.4471/rasp.2013.04

Heckhausen, J. y Schulz, R. (1995). A life span theory of control. Psychological Review, 102(2), 284-304. 


\section{López, Navarro \& Requena-Actividades de Ocio}

House, J. S., Landis, K. R. y Umberson, D. (1988). Social relationships and health. Science, 241(4865), 540-545.

Keyes, C., Ryff, C. y Shmotkin, D. (2002). Optimizing well-being: the empirical encounter of two traditions. Journal of Personality and Social Psychology, 82(6), 1007-1022.

http://dx.doi.org/10.1037/0022-3514.82.6.1007

Kirkwood, T. (1996). Mechanisms of Ageing. En Ebrahim, S. and Kalache, A. (eds.), Epidemiology in Old Age. London: BMJ Publishing Group. Merino, I. A. y García, M. L. (2005). Ocio en los mayores: calidad de vida. En: Envejecimiento, salud y dependencia. Universidad de La Rioja (pp. 43-62).

Minkler, M. y Fadem, P. (2002). Successful aging: A disability perspective. Journal of Disability Policy Studies, 12(4), 229-235.

doi: 10.1177/104420730201200402

Mortimer, J. T. y Shanahan, M.J. (2003). Handbook of the life course. New York: Plenum.

Mroczek, D. K. y Kolarz, C.M. (1998). The effect of age on positive and negative affect: A developmental perspective on happiness. Journal of Personality and Social Psychology, 75, 1333-1349.

Neugarten, B., Havighurst R. y Tobin S. (1961). The Measurement of Life Satisfactons. Journal of Gerontology, 16, 134-143.

Nimrod, G. (2007). Retirees' leisure: activities, benefits, and their contribution to life satisfaction. Leisure Studies, 26(1), 65-80. doi: 10.1080/02614360500333937

OMS (2002). Envejecimiento activo: un marco político. Revista Española de Geriatría y Gerontología, 37(S2), 74-105.

Ryan, R. M. y Deci, E. L. (2001). On happiness and human potentials: A review of research on hedonic and eudaimonic wellbeing. Annual Review of Psychology, 52, 141-66.

Ryff, C. (1989a). Beyond Ponce de Leon and life satisfaction: New directions in quest of successful aging. International Journal of Behavioral Development, 12(1), 35-55.

doi: $10.1177 / 016502548901200102$

Ryff, C. (1989b). Happiness is everything, or is it? Explorations on the meaning of psychological well-being. Journal of Personality and 
Social Psychology, 57(6), 1069-1081.

http://dx.doi.org/10.1037/0022-3514.57.6.1069

Ryff, C. y Keyes, C. (1995). The structure of psychological well-being revisited. Journal of Personality and Social Psychology, 69(4), 719727. http://dx.doi.org/10.1037/0022-3514.69.4.719

Ryff, C. y Singer, B. (1998). The contours of positive health. Psychological Inquiry, 9(1), 1-28. doi: 10.1207/s15327965pli0901_1

Ryff, C. y Singer, B. (2002). From social structure to biology. En C. Snyder y A. López (eds.), Handbook of positive psychology. Londres:

Oxford University Press (pp. 63-73).

Serrano, G. P. (ed.) (2006). ¿Cómo intervenir en personas mayores? Madrid: Editorial Dykinson.

Verghese, J., Lipton, R. B., Katz, M. J., Hall, C. B., Kuslansky, G., Derby, C.A., Ambrose, A. F., Sliwinski, M. y Buschke, H. (2003). Leisure activities and the risk of dementia in the elderly. New England Journal Medicine, 348, 2508-2516. doi: 10.1056/NEJMoa022252 Villar, F., Triadó, C., Resano, C. S. y Osuna, M. J. (2003). Bienestar, adaptación y envejecimiento: cuando la estabilidad significa cambio. Revista Multidisciplinar de Gerontología, 13(3), 152-162.

Willis, S. y Marsiske, M. (1990). Life-span perspective on practical intelligence. En D. Tupper y K. Cicerone (eds.), The Neuropsychology of Everyday Life. Boston: Kluwer Academic Publishers (pp. 183-198).

Zamarrón, M. D. (2006). El bienestar subjetivo en la vejez. Madrid: Informes Portal Mayores, 52. Lecciones de Gerontología II.

Verónica López Fernández es Profesora Adjunta en la Facultad de Educación de la Universidad Internacional de La Rioja (España)

Enrique Navarro es Profesor en la Facultad de Educación de la Universidad Internacional de La Rioja (España)

Carmen Requena Hernández es Profesora de Psicología en la Universidad de León (España)

Contact Address: vlofer@hotmail.com 